\title{
Extracting the Frequent Item Sets by Using Greedy Strategy in Hadoop
}

\author{
Mr. B. Veerendranadh ${ }^{1}$, Mr.M. Naveen Kumar $^{2}$ \\ ${ }^{l} M$. Tech in Dept. of Computer Science and Engineering, LBRCE College, Mylavaram, India. \\ ${ }^{2}$ Assistant professor in Dept. of Computer Science and Engineering, LBRCE College, Mylavaram, India.
}

\begin{abstract}
Information mining came into the presence because of mechanical advances in numerous various controls. As it were, every one of the information on the planet are of no incentive without components to proficiently and successfully remove data and learning from them. In contrast with other information mining fields, visit design mining is a generally late improvement. This paper exhibits a novel approach through which the Apriori calculation can be progressed. The adjusted calculation presents elements time devoured in exchanges filtering for competitor itemsets and the quantities of tenets produced are additionally diminished.

Catchphrases: Apriori, Frequent - itemsets, Minimum Support, Confidence, Greedy Method.
\end{abstract}

\section{Introduction}

Information mining is the way toward dissecting information from alternate points of view and compressing it into valuable data - data that can be utilized to either upgrade benefits, cuts costs, or both. Information mining has worried about a lot of consideration in the data business and in the public eye as whole as of late, because of the wide preventability of a lot of information and the pending requirement for shaping such information into valuable data and learning. Itemset mining is an exploratory information digging procedure generally utilized for finding profitable connections among information [1]. Visit itemsets mining is a center part of information mining and varieties of affiliation investigation, similar to affiliation lead mining and consecutive example mining. In visit itemsets are created from enormous or colossal informational indexes by applying a few standards or affiliation govern mining calculations like Partition strategy, Apriori procedure, Incremental, Border calculation Pincer-Search, and various different methods that set aside bigger registering opportunity to register all the continuous itemsets. Extraction of continuous itemsets is a center stride in numerous affiliation investigation systems. An itemset is known as incessant in the event that it displays in a sufficiently extensive segment of the dataset. This incessant event of thing is communicated as far as the bolster number. Subsequently, it needs confused procedures for covering up or transforming clients' private data amid an information gathering process. Additionally, these systems ought not surrender the rightness of mining comes about [2]. For instance some basic words or data that rehashed as often as possible in an informational index can be dealt with as incessant itemset for that informational index. For instance, purchasing an advanced camera taken after by Akash tablet and afterward a memory card, in the event that it happens frequently in a shopping database. It is known as (visit) consecutive example. Correspondingly substructure is alluding to different basic structures, similar to sub-trees, sub-diagrams or sub-cross sections, which might be jointed with itemsets or subsequences. On the off chance that a substructure happens repetitively, it is known as a (visit) organized example. Revelation such regular example assumes an essential part in mining relations, connections, and numerous other engaging connections alongside information. Moreover, it helps in information grouping, characterization and other information mining undertakings too [3]. Be that as it may, fundamentally less consideration has been paid to mining of occasional itemsets, despite the fact that it has obtained noteworthy utilization in

(i) mining of negative affiliation rules from occasional itemsets [4],

(ii) measurable exposure hazard appraisal where uncommon examples in unknown evaluation information can prompt factual divulgence [5],

(iii) misrepresentation recognition where uncommon examples in monetary or impose information may propose strange action related with deceitful conduct [5], and

(iv) bioinformatics where uncommon examples in microarray information may propose hereditary scatters [5]. The vast group of successive itemset mining calculations can be extensively characterized into two classes: first is hopeful generationand-test worldview and second is design development worldview. According to past examinations, it has been uncovered tentatively that example development construct calculations are computationally quicker with respect to thick datasets [6]. Examples that are once in a while found in database are regularly thought to be uninteresting and are dispensed with utilizing the bolster measure. Such examples are known as rare examples. A rare example is an itemset or a manage whose support is not exactly the minsup limit. In spite of the fact that a greater part of rare examples are uninteresting, some of them may be valuable to 
the investigation, especially those that compare to negative connections in information. Some occasional examples may likewise propose the event of intriguing uncommon occasions or outstanding circumstances in the information. For instance, if $\{$ Fire $=$ Yes $\}$ is visit yet $\{$ Fire $=$ Yes, Alarm $=$ On $\}$ is occasional, at that point the last is a fascinating rare example since it might show flawed alert frameworks. To recognize such strange circumstances, the normal support of an example must be resolved, so that, if an example ends up having an impressively bring down support than anticipated, it is proclaimed as a fascinating rare example. Mining occasional examples is a testing attempt on the grounds that there are a huge number of such examples that can be gotten from a known informational collection. All the more solely, the key issues in mining occasional examples are:

(1) how to recognize fascinating rare examples, and (2) how to productively find them in expansive informational indexes. To get an alternate point of view on different sorts of fascinating rare examples, two associated originations are negative examples and contrarily connected examples. The negative itemsets and negative affiliation rules are aggregately known as negative examples. Occasional examples, negative examples, and adversely associated designs are three firmly related ideas. Albeit occasional examples and contrarily associated designs allude just to itemsets or decides that contain positive things, while negative examples allude to itemsets or decides that contain both positive and negative things.

\section{Mining Techniques For Interesting Infrequent Patterns:}

On a fundamental level, rare itemsets are given by all itemsets that are not removed by standard incessant itemset eras calculations, for example, Apriori and FP-development. Since the quantity of rare examples exponentially vast, particularly for inadequate, high dimensional information, systems produced for mining occasional examples concentrate on finding just fascinating rare examples. Mining Negative Patterns Transaction information can be binarized by enlarging it with negative things. By applying existing successive itemset era calculation, for example, Apriori on the expanded exchanges, all the negative itemsets can be determined. Such an approach is doable just if a couple of factors are dealt with as symmetric paired. Bolster Expectation Another class of systems views a rare example as fascinating just if its genuine support is extensively littler than its normal support. For contrarily corresponded designs, the normal support is figured in view of the measurable freedom presumption. Two option approaches for deciding the normal support of an example utilizing (1) an idea chain of command and (2) an area based approach known as aberrant affiliation. Bolster Expectation Based on Concept Hierarchy Objective measures alone may not be adequate to take out uninteresting rare examples. For instance, bolster bread and portable workstation phone visit things. Despite the fact that the itemset \{bread, portable PC computer\} is rare and maybe adversely related, it is not intriguing in light of the fact that their absence of support appears glaringly evident to space specialists. Hence, a subjective approach for deciding anticipated that support is required would abstain from producing such rare examples. In the former case, bread and smart phones to two totally unique item classes, which is the reason it is not astounding to find that their support is low. Bolster Expectation Based on Indirect Association Consider a couple of things, $(a, b)$, that are seldom purchased together by clients. On the off chance that an and $b$ are inconsequential things, for example, bread and DVD player, at that point their support is required to be low. On other hand, if an and $\mathrm{b}$ are connected things, at that point their support is relied upon to be high. The normal support was already processed utilizing an idea order. Here, an approach for deciding the normal support between a couple of things by taking a gander at different things usually bought together with these two things. Roundabout affiliation has numerous potential applications. In the market wicker bin space, an and $b$ may allude to figuring things, for example, desktop and smart phones. In content mining, backhanded affiliation can be utilized to distinguish equivalent words, antonyms, or words that are utilized as a part of various settings. For instance, given an accumulations of records, the word information might be by implication related with gold by means of the arbiter mining. This example proposes that the word mining can be utilized as a part of two unique settings - information mining versus gold mining.

\section{Weighted Frequent Itemsets Mining:}

Analysts have proposed weighted continuous itemset mining calculations that mirror the hugeness of things. The premier concentration of weighted continuous itemset mining is concerns fulfilling the descending conclusion things. Each weighted affiliation rules mining calculations proposed so far have been founded on the Apriori calculation. In any case, design development calculations are a great deal more effective than Apriori based calculations. A productive weighted incessant itemset mining calculation is the fundamental approach used to push weight limitations into the example development calculation and give approaches to keep the descending conclusion resources. WFIM acknowledges a rising weight requested prefix tree. The tree is crossed base up on the grounds that the past coordinating can't keep up the descending conclusion property. A support of each itemset is generally diminished as the length of an itemset is expanded, yet the weight has an abnormal trademark. 


\section{Background}

The essential mining count in perspective of connection oversee, Apriori slanted the association lead mining gathering, and it pompous other data mining fields as well. Starting late, the thought of the examination bunch has also been based on the incidental itemset mining issue, i.e., discovering itemsets whose repeat of occasion in the researched data is not precisely or comparable to a biggest edge. Standard intermittent itemset mining estimations still experience the evil impacts of their frailty to consider neighborhood thing charming quality in the midst of the mining stage. In the ordinary itemset mining issue things having a place with esteem based data are managed also. To allow isolating things in perspective of their favorable position or power inside each trade, the makers focus on discovering more helpful connection rules, i.e., the Weighted Association Rules (WAR), which consolidates weights meaning thing importance. Nevertheless, weights are exhibited just in the midst of the regulate time wander in the wake of playing out the ordinary consistent itemset mining process.

\section{Related Work:}

In as of late 2013, Luca Cagliero and Paolo Garza recommended Infrequent Weighted Itemset Mining utilizing Frequent Pattern Growth. They addresses the revelation of rare and weighted itemsets, i.e., the Infrequent Weighted Itemsets (IWIs), from value-based weighted datasets. To address this issue, the IWI-bolster measure is characterized as a weighted recurrence of event of an itemset in the examined information. Event weights are gotten from the weights related with things in every exchange by applying a given cost work. They essentially concentrates on tailing: (i) The IWI-bolster min measure, which depends on a base cost work, i.e., the event of an itemset in a given exchange is weighted by the heaviness of its slightest fascinating thing, (ii) The IWIsupport-max measure, which depends on a greatest cost work, i.e., the event of an itemset in a given exchange is weighted by the heaviness of the most intriguing thing [1]. It is imperative when managing advancement issues, least and greatest are the most normally utilized cost capacities. Thus, they are esteemed appropriate for driving the determination of a beneficial subset of occasional weighted information relationships. In particular, the accompanying issues have been tended to: 1) IWI and Minimal IWI mining driven by a most extreme IWI-bolster min edge, and 2) IWI and Minimal IWI mining driven by a greatest IWIbolster max edge. Assignment (1) involves finding IWIs and Minimal IWIs (MIWIs) which incorporate the item(s) with the minimum nearby enthusiasm inside every exchange. Errand (2) involves finding IWIs and MIWIs which incorporate item(s) having maximal neighborhood enthusiasm inside every exchange by abusing the IWI-bolster max measure. To achieve undertakings (1) and (2), they introduce two novel calculations, to be specific Infrequent Weighted Itemset Miner (IWI Miner) and Minimal Infrequent Weighted Itemset Miner (MIWI Miner), which perform IWI and MIWI mining driven by IWI-bolster limits [1]. They attempt to limit the measure of results and just maximal continuous itemsets are considered. To secure the protection, middle mining comes about are encoded utilizing hashing technique by various servers. The proposed calculation is assessed from the points of view of precision and effectiveness [2]. In same year, SoumadipGhosh et al introduced Mining Frequent Itemsets Using Genetic Algorithm. This work done with rationale of GA to enhance the situation of frequents itemsetsinformation mining utilizing affiliation govern mining. The principle advantage of utilizing GA in visit itemsets mining is to perform worldwide hunt with less time many-sided quality. This plan gives better outcomes in enormous or bigger informational index. It is additionally straightforward and effective. They had managed a testing affiliation administer mining issue of finding incessant itemsets utilizing their prescribed GA based technique. This strategy is extremely straightforward and effective one. This is effectively tried for various extensive informational collections. The outcomes got are right and proper [3]. Proficient mining of both positive and negative affiliation rules [4]. They concentrate on distinguishing the relationship among visit itemsets. They have to limit the unsafe effects and in addition boost conceivable advantages. They outlined another technique for proficiently mining both positive and negative affiliation administers in databases. This approach is novel and not quite the same as existing examination endeavors on affiliation investigation. Some rare itemsets are of enthusiasm for this strategy yet not in existing examination endeavors. They had additionally planned requirements for lessening the pursuit space, and had utilized the expanding level of the contingent likelihood in respect to the earlier likelihood to assess the certainty of positive and negative affiliation rules. Their exploratory outcomes have exhibited that the proposed approach is viable, productive and promising [4].

On insignificant rare itemset mining was proposed by D. J. Haglin and A. M. Keeping an eye on. They exhibit another calculation for finding negligible occasional examples. This is the main calculation planned particularly to find insignificant rare itemsets. The least demanding approach to depict the distinctions in dataset properties is to consider the network shape. For customary itemset mining, the network comprises of twofold passages. They can change a SUDA2-sort network into a twofold framework by identifying the majority of the sets. For each of such combines, a section is framed in the changed double framework. For each incentive in a section in the SUDA2-sort input lattice, the relating area in the changed parallel grid is given a one [5]. They suggest another calculation in light of the example development worldview to discover insignificantly rare 
itemsets. It has no subset which is additionally occasional. They additionally present the novel idea of remaining trees. Later on used the remaining trees to mine various level least support itemsets where distinctive limits are utilized for finding incessant itemsets for various lengths of the itemset. At long last, they break down the conduct of our calculation as for various parameters and show through trials [6]. They made a reasonable commitment like; they proposed another calculation IFP min for mining negligibly rare itemsets and a streamlining on the Apriori calculation to mine insignificantly rare itemsets. They present the idea of leftover trees utilizing a variation of the FP-tree structure named as backwards FPtree. They likewise exhibit a nitty gritty investigation to evaluate the effect of variety in the thickness of datasets on the calculation time of Apriori, MINIT and our calculation. They extend the proposed calculation to mine continuous itemsets in the MLMS structure [6]. Weighted regular itemset mining with a weight territory and a base weight otherwise called WFIM is proposed by Yun, Unil, and John J. Leggett in year 2005. A weight territory and a base weight limitation are characterized and things are given diverse weights inside the weight assortment. The weight and maintain of every thing are considered independently to prune the hunt space. The various weighted regular itemsets can be decreased by setting a weight territory and a base weight, allowing the client to harmony support and weight of itemsets. WFIM delivers all the more outlining and critical weighted regular itemsets in immense databases, prevalently extreme databases with low slightest support, by adjusting a base weight and a weight territory. They utilized the term, weighted itemset to speak to an arrangement of weighted things [7]. A basic approach to accomplish a weighted itemset is to compute the normal estimation of the weights of the things in the itemset. WFIM utilizes FP-trees as a pressure system. FP-trees are for the most part utilized as a part of example development calculations. WFIM figures nearby continuous things of a prefix by filtering its anticipated database. The FP-trees in our calculation are made as takes after. Output the exchange database one time and tally the support of every thing and check the heaviness of every thing. After this, sort the things in weight rising request. Despite the fact that backings of things might be lower than the base support and rare, the things can't be erased since occasional things may wind up noticeably weighted itemsets in the following stage. The broad execution examination demonstrates that WFIM is productive and adaptable in weighted continuous itemset mining. Many enhanced calculations utilizing separate and vanquish techniques have been likewise recommended [7]. In late year 2013, Diti Gupta and Abhishek Singh Chauhan displayed an overview on Mining Association Rules from Infrequent Itemsets. They study about the finding of negative and positive affiliation rules frame the occasional itemset.

They accompany a few focal points and the issue detailing which can be actualized in future. In light of this examination they recommended that to augment the support-certainty structure in a dynamic mold. Notwithstanding finding sure positive decides that have a solid correspondence, the calculation decides negative affiliation rules with solid negative connection between's the forerunners and consequents. So they can plan a capable framework which produces both positive and negative affiliation rules. They additionally create a wide range of limited tenets, accordingly allowing to be utilized as a part of various applications where every one of these sorts of guidelines could be required or only a subset of them. Thus they get better recurrence result set for the whole thing set in both positive and negative affiliations [8]. Affiliation govern mining is to discover affiliation decides that fulfill the predefined least support and certainty from a predetermined database. In the continuous circumstance they can subdivide the issue in two sections. In the first place is to locate the set surpass a predefined edge in the database; those thing sets are called successive or expansive thing sets. The second stage is the event era from affiliation rules. In this manner if applying dynamic least bolster at that point level savvy deterioration is simple. On the off chance that we think about the most fitting and productive information mining calculation then we generally consider Apriori calculation. However there are two bottlenecks of the Apriori calculation. The procedure of applicant era is first which can build the time and also the space. Along these lines the second thing is delivered from the principal that it required various sweep when it in the emphasis methodology. Established on Apriori calculation, different new calculations were composed with a few adjustments or enhancements. The computational cost of affiliation rules mining can be decreased by lessening the passes, utilizing examining and through including additional obliges according to request [8]. In 2012, YihuaZhong et al. [9] recommend that affiliation control is a vital model in information mining. Still, regular affiliation rules are generally in view of the certainty measurements and bolster, and most calculations and explores unspecified that each quality in the database is proportionate. Truth be told, since the client slant to the thing is disparate, the mining rules utilizing the offered calculations are not generally appropriate to clients. By presenting weighted double certainty, another calculation that can mine productive weighted principles is recommended by the creators. This investigation exhibit that the calculation can lessen the vast number of useless affiliation guidelines and mine intriguing negative affiliation runs, all things considered. By presenting the idea of weighted double certainty, another new calculation can mine powerful weighted guidelines that are on the premise of the double certainty affiliation rules utilized as a part of calculation. In 2012, He Jiang et al. [10] bolster the strategy that enables the clients to determine numerous base backings to mirror the natures of the itemsets and their changed frequencies in the database. This plan is extremely productive for tremendous 
databases to utilize calculation of affiliation rules in view of various backings. The introduced calculations are regularly mining positive and negative affiliation rules from visit itemsets. Barring the negative affiliation rules from rare itemsets are disregarded. Moreover, they set diverse weighted esteems for things as indicated by the significance of every thing. As per three elements specified over, a calculation for mining weighted negative affiliation rules from occasional itemsets in view of numerous backings (WNAIIMS) is proposed by the creator. They set diverse least support for itemsets. Amid affiliation control mining, if the predefined least support is too much high, at that point the things with low event of development couldn't be mined. Something else, if the predefined least support is exorbitantly low, at that point gathering blast may emerge. In 2010 Younghee Kim et al [11] gave Mining Frequent Itemsets Normalized Weight in Continuous Data Streams. They consider the issue of mining with weighted support over an information stream sliding window utilizing restricted memory space, called WSFI-Mine. WSFI-Mine stands for Weighted Support Frequent Itemsets Mine. This calculation enables the client to determine the weight for every thing. It can find valuable late information from an information stream by utilizing a solitary sweep. In view of the weighted support, we propose another calculation, to proficiently find all the regular itemsets from streams. This technique is driven by an outer weight table or weight work. The proposed WSFI-Mine technique is intended to mine all continuous itemsets from one sweep in the datastreams. They propose a WSFI-Mine that can mine powerfully kept up use designs utilizing data from a past sliding time that can be refreshed continuously. The WSFI-Mine calculation has three stages: the standardization of weight bolster and separating designs into three classifications, the development of the WSFP-Tree, and a successive itemset disclosure conspire. Development of a WSFP-Tree guarantees that incessant example mining can be performed effectively. A WSFP-Tree is an information structure in light of an expanded FP-tree. It serves to store compacted urgent data about incessant examples [11].

A Survey on Algorithms for Mining Frequent Itemsets over Data Streams was exhibited by James Cheng et al. [12] in year 2008. review various agent stateof-the-craftsmanship calculations on mining incessant itemsets, visit maximal itemsets, or successive shut itemsets over information streams. They organized the calculations into two classifications in view of the window show that they embrace: the point of interest window or the sliding window. Each window portrayal is then arranged as time-based or countbased. As per the quantity of exchanges that are refreshed each time, the calculations are further characterizing into refresh per exchange or refresh per cluster. At that point, arrange the mining calculations into two classes: correct or assessed. They additionally classified the assessed calculations as per the outcomes they restore: the false-positive approach or the false-negative approach. The false-positive approach restores an arrangement of itemsets that incorporates all regular itemsets additionally some rare itemsets, while the falsenegative approach restores an arrangement of itemsets that does exclude any occasional itemsets but rather misses some successive itemsets. They likewise talked about the diverse issues raised from the distinctive window models and the idea of the calculations. They likewise clarify the crucial standard of the ten calculations and investigate their benefits and constraints [12]. They [12] additionally centered around visit itemset mining and have attempted to cover both early and late writing identified with mining regular itemsets (FIs) or incessant shut itemsets (FCIs). Specifically, they have examined in detail some of the best in class calculations on mining FIs, FMIs or FCIs over information streams. In addition, we have tended to the benefits and the impediments and introduced a general investigation of the calculations, which can give bits of knowledge to end-clients in applying or building up a proper calculation for various gushing conditions and different applications [12]. In 2012, IdhebaMohamad Ali O. Swesiet al. [13] examine is to build up another model for mining fascinating negative and positive affiliation precludes of a value-based informational index. Their proposed show is reconciliation between two calculations, the Positive Negative Association Rule (PNAR) calculation and the Interesting Multiple Level Minimum Supports (IMLMS) calculation, to propose another approach (PNAR_IMLMS) for mining both negative and positive affiliation rules from the fascinating regular and rare thing sets mined by the IMLMS portrayal.

The investigational comes about show that the PNAR_IMLMS demonstrate gives essentially preferred outcomes over the past model. In 2009, Yuanyuan Zhao et al. [14] recommend that the Negative affiliation rules turn into a concentration in the information mining field. Negative affiliation rules are useful in advertise wicker bin examination to distinguish items that contention with each other or items that backup each other. The negative affiliation controls every now and again comprise in the rare things. The examination demonstrates that the quantity of the negative affiliation rules from the occasional things is bigger than those from the incessant. This investigation centered to actualize the Global Profit Weight (GPW) for the continuous thing set. For the most part the benefit can be measured generally. In this examination they proposed multi criteria based benefit figuring. They additionally talked about the significant issue is to mine the affiliation rules with weighted things, in view of the diverse sorts of the affiliation rules, which are double affiliation rules and quantitative affiliation rules. New calculations are required to take care of such issues since the accessible calculations can't be explained. The goal of study is to actualize the worldwide benefit weight measure and test the execution of the calculation with conventional weighted affiliation lead mining. The usage is communicated as step savvy visual introduction and its execution is measured with weighted ARM. The exactness is characterized utilizing 
characterization calculation, for example, Navie Bayes, VFI, BF Tree and IB1and the outcomes are thought about utilizing WEKA. It can be finished up from the investigation result that GPW is required high calculation energy to create the weight. The outcome is traded off with its quality. As indicated by the exploration issue, the computed weight can be reused it for commonly and as required [15]. This work utilizes worldwide benefit weight calculation is actualized utilizing visual essential to discover the benefit of the thing set in the exchange. Arrangement calculation is utilized to assess the benefit measure, for example, high $(\mathrm{H})$, medium $(\mathrm{M})$ and low (L). Generally utilized directed machine learning methods specifically Naïve Bayes Decision tree classifier, VFI and IB1 Classifier are used for taking in the portrayal. The results of the models are assessed and watched that Naive Bayes performs well. The venture sums up past work on benefit measure. The benefit measure of the things in the exchange is characterized by utilizing the normal for the thing. Considering the benefit of a thing, there are various vital variables to consider also. They concentrate on the mining of weighted affiliation rules for which the heaviness of a thing set is standardized by the span of the thing set. The decision of utilizing disorderly or standardized weight was relied on upon the individual need of every application [15].

\section{PROBLEM STATEMENT}

The strategy actualized here for the mining of occasional weighted thing sets gives less execution time and contains less capacity and the quantity of hubs made are likewise less on the premise of support and certainty. Be that as it may, future improvements should be possible as to incorporate the proposed approach in a propelled basic leadership framework that backings area master's focused on activities in light of the qualities of the found IWIs. Moreover, the utilization of various total capacities other than least and most extreme will be examined.

\section{PROPOSED PLAN}

1. Information dataset.

2. Pass Support and certainty on the premise of which least support is computed.

3. Apply Association govern digging calculation for the era of successive sets and affiliation rules.

4. Order visit and occasional thing sets utilizing Greedy.

\section{GREEDY ALGORITHM}

Orthogonal coordinating interest (OMP) calculation has gotten much consideration as of late. OMP calculation is an iterative voracious calculation that chooses at each progression the section. Orthogonal coordinating interest (OMP) develops an estimate by experiencing an emphasis procedure. At every cycle the locally ideal arrangement is figured. This is finished by finding the segment vector in A which most nearly looks like a leftover vector $r$. The lingering vector begins being equivalent to the vector that is required to be approximated i.e. $\mathrm{r}=\mathrm{b}$ and is balanced at every cycle to consider the vector already picked. The expectation this grouping of locally ideal arrangements will prompt the worldwide ideal arrangement. As normal this is not the situation when all is said in done in spite of the fact that there are conditions under which the outcome will be the ideal arrangement. OMP depends on a variety of a prior calculation called Matching Pursuit (MP). MP essentially expels the chose section vector from the lingering vector at every emphasis. $\mathrm{rt}=\mathrm{rt}-1$ - rt-1 Where aOP is the segment vector in A which most nearly takes after rr-1.OMP utilizations a minimum squares venture at every emphasis to refresh the lingering vector with a specific end goal to enhance the guess. The OMP is a stepwise forward choice calculation and is anything but difficult to actualize.

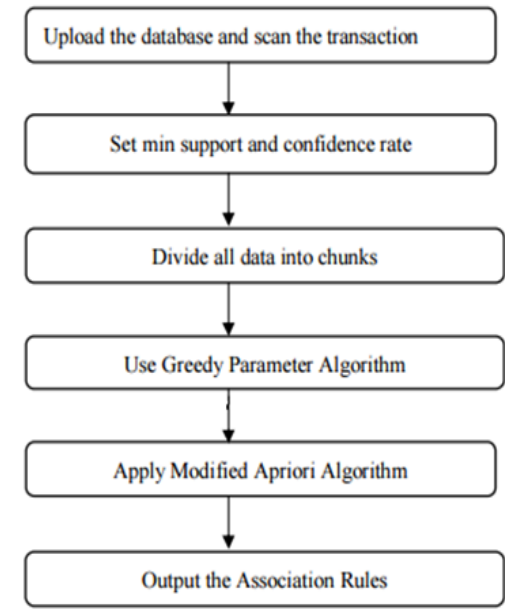

Fig:1 Greedy Algorithm 
A voracious calculation is a numerical procedure that searches for straightforward, simple to-execute answers for complex, multi-step issues by choosing which subsequent stage will give the most evident advantage. Such calculations are called avaricious in light of the fact that while the ideal answer for each littler example will give a prompt yield, the calculation doesn't consider the bigger issue all in all. Once a choice has been made, it is never reevaluated. Covetous calculations work by recursively building an arrangement of articles from the littlest conceivable constituent parts. Recursion is a way to deal with critical thinking in which the answer for a specific issue relies on upon answers for littler cases of a similar issue. The preferred standpoint to utilizing an avaricious calculation is that answers for littler occasions of the issue can be clear and straightforward.

\author{
Algorithm greedyAlg1 \\ Input: $\quad D \quad / /$ the categorical database \\ $k \quad / /$ the number of desired items \\ Output: $k$ identified items

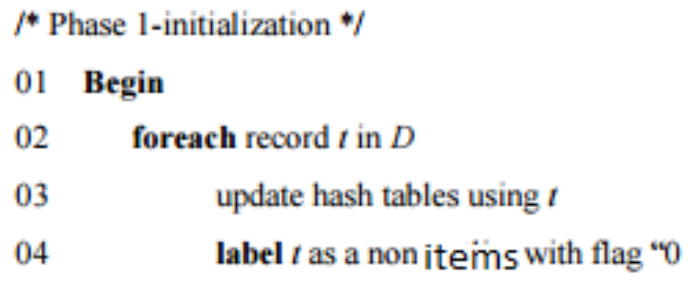

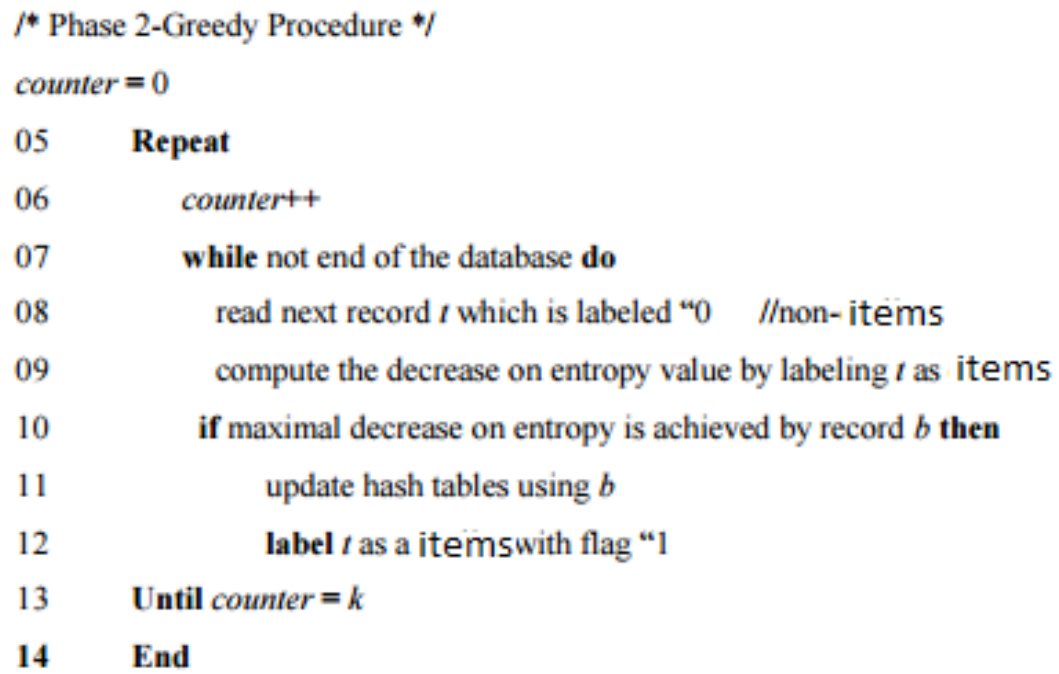

\title{
References
}

[1] Luca Cagliero and Paolo Garza "Infrequent Weighted Itemset Mining using Frequent Pattern Growth", IEEE Transactions on Knowledge and Data Engineering, pp. 1- 14, 2013.

[2] Xin Li, XuefengZheng, Jingchun Li, Shaojie Wang "Frequent Itemsets Mining in Network Traffic Data", 2012 Fifth International Conference on Intelligent Computation Technology and Automation, pp. 394- 397, 2012.

[3] SoumadipGhosh, SushantaBiswas, DebasreeSarkar, ParthaPratimSarkar "Mining Frequent Itemsets Using Genetic Algorithm", International Journal of Artificial Intelligence \& Applications (IJAIA), Vol.1, No.4, pp. 133 - 143, October 2010.

[4] X. Wu, C. Zhang, and S. Zhang "Efficient mining of both positive and negative association rules", ACM Transaction Information System, vol. 22, issue 3, pp. 381-405, 2004.

[5] D. J. Haglin and A. M. Manning "On minimal infrequent itemset mining”, In DMIN, pp. 141-147, 2007.

[6] Ashish Gupta, Akshay Mittal and Arnab Bhattacharya "Minimally Infrequent ItemsetMining using Pattern-Growth Paradigm and Residual Trees", 17th International Conference on Management of Data (COMAD), 2011.

[7] Yun, Unil, and John J. Leggett. "WFIM: weighted frequent itemset mining with a weight range and a minimum weight", In Proceedings of the Fifth SIAM International Conference on Data Mining, pp. 636 - 640, 2005.

[8] Diti Gupta and Abhishek Singh Chauhan "Mining Association Rules from Infrequent Itemsets: A Survey", International Journal of Innovative Research in Science, Engineering and Technology, ISSN: 2319-8753, Vol. 2, Issue 10, pp. 5801 - 5808, 2013

[9] YihuaZhong; Yuxin Liao, "Research of Mining Effective and Weighted Association Rules Based on Dual Confidence," Fourth International Conference on Computational and Information Sciences (ICCIS), pp.1228 - 1231, Aug. 2012. 
[10] He Jiang, Xiumei Luan and Xiangjun Dong," Mining Weighted Negative Association Rules from Infrequent Itemsets Based on Multiple Supports", International Conference on Industrial Control and Electronics Engineering, pp. 89 - 92, 2012.

[11] Younghee Kim, Wonyoung Kim and Ungmo Kim "Mining Frequent Itemsets with Normalized Weight in Continuous Data Streams", Journal of Information Processing Systems, Vol.6, No.1, March 2010

[12] James Cheng, YipingKe, and Wilfred Ng “A Survey on Algorithms for Mining Frequent Itemsets over Data Streams”, Knowledge and Information Systems, Volume 16, Issue 1, pp. 1 - 27, July 2008.

[13] IdhebaMohamad Ali O. Swesi, Azuraliza Abu Bakar, AnisSuhailis Abdul Kadir, "Mining Positive and Negative Association Rules from Interesting Frequent and Infrequent Itemsets", 9th International Conference on Fuzzy Systems and Knowledge Discovery, pp. $650-655,2012$

[14] YuanyuanZhao,He Jiang; RunianGeng; Xiangjun Dong, "Mining Weighted Negative Association Rules Based on Correlation from Infrequent Items," Advanced Computer Control,ICACC '09. International Conference on, vol., no., pp.270 - 273, 22-24 Jan. 2009.

[15] AshaRajkumar and G.SophiaReena "Frequent Item set Mining Using Global Profit Weight Algorithm", International Journal on Computer Science and Engineering, Vol. 02, No. 08, pp. 2519-2525, 2010

IOSR Journal of Computer Engineering (IOSR-JCE) is UGC approved Journal with Sl. No. 5019, Journal no. 49102.

Mr. B. Veerendranadh. "Extracting the Frequent Item Sets by Using Greedy Strategy in Hadoop." IOSR Journal of Computer Engineering (IOSR-JCE) 19.4 (2017): 83-90. 